\title{
Characterization of the Burst Aggregation Process in Optical Burst Switching
}

\author{
Xenia Mountrouidou and Harry G. Perros \\ Computer Science Department, \\ North Carolina State University, \\ Raleigh, NC 27695, USA \\ \{pmountr, hp\}@csc.ncsu.edu
}

\begin{abstract}
We describe an analytic approach for the calculation of the departure process from a burst ggregation algorithm that uses both a timer and maximum/minimum burst size. The arrival process of packets is assumed to be Poisson or bursty modelled by an Interrupted Poisson Process (IPP). The analytic results are approximate and validation against simulation data showed that they have good accuracy.
\end{abstract}

\section{Introduction}

An important design aspect of an OBS network is the burst aggregation process performed at the edge nodes. This process concentrates upper layer packets which are then transmitted optically over the OBS network. In view of this, the burst aggregation strategy defines the burst arrival process to the OBS network. This process depends on the parameters of the aggregation process, and so far it has not been adequately studied. However, it is important that the burst arrival process to an OBS network is well characterized if we are to understand better the performance of OBS networks.

The main parameters of a burst assembly algorithm are a timer and the maximum and minimum burst size. When the timer expires, the edge node assembles a burst that consists of packets in the edge's packet queue that have the same destination. This procedure takes place in the electrical domain, and the resulting bursts are transmitted in the optical domain. If the arrival rate at an edge node is very high, then each time the timer expires, there may be a large number of packets waiting in the packet queue. This would lead to large data bursts if all packets are assembled into a single burst. Large bursts decrease the performance in the OBS network, since they occupy the resources for long intervals. As a result, they block other bursts thus leading to a high burst loss probability [1]. In order to avoid this problem, a maximum burst size is used to bound the size of a burst. Another drawback of the burst assembly algorithm, if it is driven entirely by a timer, is that bursts can be very small if the arrival rate to the edge node is very low. This results to high overheads, since the OBS network has to set up a path for each burst. The solution to this problem is to use a lower bound for the burst size. If this lower limit is not reached, when the timer expires, then the burst is not transmitted. 
Various algorithms have been proposed to aggregate packets into bursts. Most of these assembly algorithms use either an assembly timer or a maximum and minimum burst length or both as a way of creating bursts. Let $T$ be the length of the timer, $B_{\max }$ the maximum burst length and $B_{\min }$ the minimum burst length. We can classify the assembly algorithms into the following three categories:

- Time-based aggregation algorithms: In this case a fixed-threshold $T$ is used to create a burst. In some implementations, a minimum length $B_{\text {min }}$ is required 22. If the burst is shorter than $B_{\text {min }}$ then padding is used to increase the length to $B_{\text {min }}$. The shortcoming of the time-based assembly algorithm is that, under heavy traffic load, the number of packets that are gathered until the timer expires may be high, thus resulting to large bursts.

- Burst-length based aggregation algorithms: In this case, the burst is sent out as soon as the burst length exceeds a given maximum burst length $B_{\max }$. Thus, the packets are buffered until the total size reaches the maximum threshold. The main disadvantage of this algorithm is that it does not constraint the waiting time of the packets in the packet queue. Therefore, when the traffic is low, waiting time may be large.

- Time and burst-length based burst aggregation algorithms: The disadvantages of the aggregation algorithms based on a timer or a maximum burst length can be overcome using a combination of a timer and maximum and minimum burst lengths. In this case, the packets are buffered until the timer expires. Then, we compare the total size of the packets in the queue with the upper and lower limits, $B_{\max }$ and $B_{\min }$. If the size is less than $B_{\min }$, then we keep the packets in the packet queue until the next aggregation period, i.e. until the next time when the timer expires. If the size is greater than $B_{\min }$ but less than $B_{\max }$ we aggregate all the packets in one burst. If the size is greater than $B_{\max }$, we make one burst of maximum size and then we repeat this process with the remaining bits.

We note that an adaptation scheme has to be created which will assemble packets into bursts at the transmitter's side, and correctly recover these packets at the receiver's side. There are several examples of adaptation schemes, such as the schemes used for the formation of AAL 5 PDU and AAL 2 CPS packets in ATM networks. In OBS depending upon the adaptation scheme, a packet may straddle over two successive bursts. Alternatively, a burst may be allowed to exceed a maximum burst size, so that the last packet is included in its entirety. In this paper we assume that the maximum burst size is strictly enforced, and therefore a packet may straddle over two successive bursts.

The burst aggregation process has been studied in [3], 4], [5], 6] and [2]. Papers [4, 5], 6] and [2] study the effect of burst aggregation algorithms on the self-similarity characteristics of the input traffic. 3] gives an analytical method to calculate the aggregated burst size for various algorithms, and assuming Poisson arrivals of packets to the edge node. The authors did not consider the aggregation algorithm that uses both a timer and a maximum/minimum burst size analyzed in this paper. In this paper we obtain analytically the distribution of the number of bursts created by the aggregation algorithm which uses both a timer and a 
maximum/minimum burst size. We first assume that the arrival of packets to the edge node is Poisson, and then we extend the analysis to the case where packets arrive according to an Interrupted Poisson Process (IPP). This process models bursty traffic such as video, voice or data.

The rest of this paper is organized as follows. In Section 2 we obtain analytically the distribution of the number of bursts created at each aggregation period assuming Poisson arrivals. In Section 3 we extend these results to IPP arrivals. The results obtained in this paper are approximate and in Section 4 we compare our analytical results with simulation data. Finally, Section 5 gives the conclusions.

\section{The Case of Poisson Arrivals}

We consider an edge node which receives packets in the electronic domain and transmits them to destination edge nodes optically over an OBS network. The arriving packets are queued to different packet queues, each associated with a different destination edge node. We only consider a single packet queue in which packets are queued for a specific destination. We assume that the arrival process of packets to the queue is Poisson with a rate of $\lambda$. Packet size is exponentially distributed with a mean of $1 / b$ bytes. We recall that the length of the aggregation period, i.e. the time after which the timer expires, is $T$.

Since packets arrive in a Poisson fashion, the probability that $n$ packets arrive within $T$ is: $P[X=n]=e^{-\lambda T} \frac{(\lambda T)^{n}}{n !}$. Therefore, the pdf of the number of bytes $B$ that arrive during the $i^{\text {th }}$ aggregation period $((i-1) T, i T]$ is:

$$
f_{B}(x)=\sum_{n=1}^{\infty} P[X=n] f_{S_{n}}(x),
$$

where $f_{S_{n}}(x)$ is the probability that the total number of bytes associated with $n$ packets is $x$. This is obtained by convoluting $n$ i.i.d. exponentially distributed variables, which in fact is the pdf of an n-stage Erlang distribution [7], given by: $f_{S_{n}}(x)=\frac{b(b x)^{n-1} e^{-b x}}{(n-1) !}$. Thus, the pdf of the number of bytes that arrive during the $i^{\text {th }}$ aggregation period is:

$$
f_{B}(x)=\sum_{n=1}^{\infty} \frac{e^{-\lambda T}(\lambda T)^{n} b(b x)^{n-1} e^{-b x}}{n !(n-1) !}
$$

The cumulative distribution function (cdf) of the number of bytes in the packet queue at the end of the period $T$ is:

$$
F_{B}(x)=\int_{0}^{\infty} f_{B}(x)
$$

where $f_{B}(x)$ is given by $(2)$. Using $F_{B}(x)$ we can calculate the probability of the number of bursts that are formed at the end of each period $T$. For instance, the 
number of bytes $x$ has to be within the interval $\left[0, B_{\min }-1\right]$ in order to have zero bursts, it has to be within $\left[B_{\min }, B_{\min }+B_{\max }-1\right]$ in order to have one burst, and in general it has to be within the interval $\left[B_{\min }+(k-1) B_{\max }, B_{\min }+k B_{\max }-1\right]$ in order to have $k$ bursts. That is:

$$
\begin{gathered}
P[k=0 \text { bursts }]=\int_{0}^{B_{\min }-1} f_{B}(x) \\
P[k \text { bursts }]=\int_{B_{\min }+(k-1) B_{\max }}^{B_{\min }+k B_{\max }-1} f_{B}(x), k>=1
\end{gathered}
$$

Expression 2 is quite difficult to work with, and whenever possible it is approximated by a simple mixture of exponential distribution as described below. As will be seen in order to do this, we need the first three moments of the number of bytes in the packet queue at the end of each aggregation period. We note that the number of bytes that arrive during period $T$ is a random sum of exponentially distributed variables. Therefore, the moment generating function (MGF) of the number of bytes is [8]:

$$
M_{B}(t)=M_{N}\left(\ln \left(M_{S}(t)\right)\right)
$$

where $M_{B}(t)$ is the MGF of the number of bytes during interval $((i-1) T, i T]$, $M_{N}(t)$ is the MGF of the number of packets $N$, and $M_{S}(t)$ the MGF of the packet size $S$. Thus, we have: $M_{N}(t)=e^{\lambda T\left(e^{l n\left(M_{S}(t)\right)}-1\right)}, M_{S}(t)=\frac{b}{b-t}$ and therefore:

$$
M_{B}(t)=e^{\lambda T\left(\frac{b}{b-t}-1\right)}
$$

At the end of each aggregation period there may be a residual number of bytes $r$ which are not transmitted in a burst because of the condition that a burst has to be at least greater than $B_{\min }$. We have found empirically that if $B_{\min }<<<$ $B_{\max }$, then the residual number of bytes is typically zero. On the other hand, if $B_{\min }$ is close to $B_{\max }$, then we have observed that the residual number of bytes is uniformly distributed within $\left[0, B_{\min }\right)$. For instance, if $B_{\min }=16$ Kbytes and $B_{\max }=112$ Kbytes, then there is a high probability that the last burst will be larger than 16 Kbytes, which means that the residual number of bytes will be zero. However, if we set $B_{\min }=85 \mathrm{Kbytes}$ then there is a high probability that the last burst will not be greater than $B_{\text {min }}$, which means that it will not be transmitted out. This remainder can be safely assumed that it is uniformly distributed within $\left[0, B_{\min }\right)$.

In view of the above empirical observations, we distinguished two cases. If $B_{\min }<<<B_{\max }$, then we assume that there is zero left over bytes from the previous aggregation period, in which case the pdf of $f_{B}(x)$ and its MGF $M_{B}(t)$ are given by expressions (4) and (8). If $B_{\min }$ is close to $B_{\max }$, then the pdf $f_{X}(x)$ of the number of bytes at the end of an aggregation period is given by the convolution of $f_{B}(x)$ and $f_{r}(x)$. We have: $f_{X}(x)=f_{B}(x) * f_{r}(x)$. Therefore, the MGF of $f_{X}(x), M_{X}(t)$ is given by $([9])$ :

$$
M_{X}(t)=M_{B}(t) M_{r}(t) \text { or } M_{X}(t)= \begin{cases}e^{\lambda T\left(\frac{b}{b-t}-1\right)} \frac{e^{t B_{\min }}-1}{t B_{\min }} & \text { if } t>0 \\ e^{\lambda T\left(\frac{b}{b-t}-1\right)} & \text { if } \mathrm{t}=0\end{cases}
$$


We can now calculate the moments of the distribution of the number of bytes available in an aggregation period for both models. In the first model, where we do not include the residual number of bytes, we have:

$$
\begin{gathered}
m_{1}=M_{B}^{\prime}(0)=\frac{\lambda T}{b} \\
m_{2}=M_{B}^{\prime \prime}(0)=\frac{\lambda T}{b^{2}}(2+\lambda T) \\
m_{3}=M_{B}^{(3)}(0)=\frac{\lambda T}{b^{3}}[(2+\lambda T)(3+\lambda T)+\lambda T]
\end{gathered}
$$

In the second model, where we include the residual of the number of bytes, we have:

$$
\begin{gathered}
m_{1}=M_{X}^{\prime}(0)=\frac{\lambda T}{b}+\frac{B_{\text {min }}}{2} \\
m_{2}=M_{X}^{\prime \prime}(0)=\frac{\lambda T}{b^{2}}(2+\lambda T)+\frac{B_{\text {min }}^{2}}{3}+\frac{\lambda T B_{\text {min }}}{b} \\
m_{3}=M_{X}^{(3)}(0)=\frac{\lambda T}{b^{3}}[(2+\lambda T)(3+\lambda T)+\lambda T]+\frac{B_{\text {min }}^{3}}{4}+\frac{3 \lambda T B_{\text {min }}}{b}\left[\frac{1}{2 b}(2+\lambda T)+\frac{B_{\text {min }}}{3}\right]
\end{gathered}
$$

From the first three moments of these different models, we see that the number of bytes that arrive within a period $T$ and the residual from the previous period are independent. This is because of the way we calculated the total number of bytes available at the end of period $T$.

Using the three moments, we can now approximate the pdf $f_{B}(x)$ or $f_{X}(x)$ of the total number of bursts in the packet queue at the end of a period $T$ by a twostage Coxian, $C_{2}$ [10. For this we set the first three moments, $m_{1}, m_{2}, m_{3}$ equal to the first three moments of $C_{2}$ with parameters $\left(\mu_{1}, \mu_{2}, \alpha\right)$. The three moment fit, can be used if $3 m_{2}^{2}>2 m_{1} m_{3}$ and $c^{2}>1$, where $c^{2}$ is the squared coefficient of variation. Alternatively a two moment fit can be used if the condition $3 \mathrm{~m}_{2}^{2}>$ $2 m_{1} m_{3}$ does not hold or $0.5<c^{2}<1$. The pdf of a $C_{2}$ is given by the expression:

$$
f_{Y}(y)=(1-\alpha) \mu_{1} e^{-\mu_{1} y}+\alpha\left(\frac{\mu_{1} \mu_{2}}{\mu_{2}-\mu_{1}} e^{-\mu_{1} y}+\frac{\mu_{1} \mu_{2}}{\mu_{1}-\mu_{2}} e^{-\mu_{2} y}\right)
$$

where in the case of the three-moment fit: $\mu_{1}=\frac{L+\left(L^{2}-4 K\right)^{1 / 2}}{2}, \mu_{2}=L-$ $\mu_{1}$ and $\alpha=\left(\left(\mu_{1} m_{1}\right)-1\right), \quad K=\frac{6 m_{1}-3\left(m_{2} / m_{1}\right)}{\left(\left(6 m_{2}^{2}\right) /\left(4 m_{1}\right)-m_{3}\right.}, L=1 / m_{1}+\frac{m_{2} K}{2 m_{1}}$ and in the case of the two-moment fit: $\mu_{1}=\frac{2}{\mu_{1}}, \mu_{2}=\frac{1}{\mu_{1} c^{2}}$ and $\alpha=\frac{1}{2 c^{2}}$. Using the $C_{2}$ pdf we can easily calculate the cumulative distribution $F_{X}(x)$ and from there the probability of creating $k$ bursts at the end of each period $T$ (see equations [3. 4).

When $c^{2}<0.5$, we can fit an Erlang distribution or a generalized Erlang distribution (see [10]). However we observed empirically, that the number of bytes 
in the packet queue at the end of an aggregation period has a small variability. In view of this, we have found that it is sufficient to evaluate $f_{B}(x)$, given by equation 2, for only a small range of values of $n$. That is, we limit the sum to:

$$
f_{B}(x)=\sum_{n=\text { avgNumPacks }-10 \sigma}^{\operatorname{avgNumPacks+10\sigma }} \frac{e^{-\lambda T}(\lambda T)^{n} b(b x)^{n-1} e^{-b x}}{n !(n-1) !}
$$

where $\operatorname{avgNumPacks}=\lambda T$, is the average number of packets that arrive during a period $T$, and $\sigma=\sqrt{\lambda T}$ is the variance of the number of packets that arrive during $T$. From 15 we can then numerically compute the cumulative distribution of the pdf $f_{B}(x)$ or $f_{X}(x)$ and subsequently the probability of having $k$ bursts, where $k \geq 0$ (see equations 3, 4).

Due to the limited variability of the number of bytes in $T$, we have also found that the following approximation gives good results:

$$
P[k \text { bursts }]=\frac{m_{1} / B_{\max }}{\left\lceil m_{1} / B_{\max }\right\rceil}, P[(k-1) \text { bursts }]=1-P[k \text { bursts }]
$$

where $k=m_{1} / B_{\max }$.

\section{The Case of IPP Arrivals}

The IPP is a modified Poisson process. It is similar to a Markov Modulated Poisson Process with two states (MMPP2). The main difference between IPP and MMPP2 is that the arrival rate in the second state of the MMPP2 is zero, which means there are no arrivals in this state [1]. An IPP is an ON/OFF process, where the ON and OFF periods are exponentially distributed with rates $\sigma_{1}$ and $\sigma_{2}$ respectively. We also define the vector $\pi: \boldsymbol{\pi}=\left(\pi_{1}, \pi_{2}\right)=\frac{1}{\sigma_{1}+\sigma_{2}}\left(\sigma_{2}, \sigma_{1}\right)$ which gives the average duration of the ON and OFF periods. During the ON period there are Poisson arrivals with rate $\lambda$, and during the OFF period there are no arrivals. This is a very useful model for data/voice and video transfers over the Internet, where bursty arrivals of packets occur for a period of time followed by an idle interval. We assume that the packet sizes are exponentially distributed with an average size $1 / b$ bytes. The IPP process is also characterized by the squared coefficient of variation, $c_{I P P}^{2}$, of the packet interarrival time, that measures the burstiness of the arrival process, given by the expression: $c_{I P P}^{2}=1+\frac{2 \lambda \sigma_{1}}{\left(\sigma_{1}+\sigma_{2}\right)^{2}}$. From this equation we can observe that the value of $c_{I P P}^{2}$ is affected by the duration of the $\mathrm{ON}$ and OFF periods. We also define the quantity: average transmission rate $=$ transmissionSpeed $\frac{\sigma_{2}}{\sigma_{1}+\sigma_{2}}$ The average transmission rate depends on the transmission speed, it is in fact the transmission speed within the ON period. In our implementation, we set $1 / \lambda=\frac{\text { average packet size }}{\text { transmissionspeed }}=\frac{1 / b}{10 \text { Gbps }}$, where $1 / b=500$ bytes. Thus, $1 / \lambda$ is the time needed to transmit one packet during the ON period. Given the $c_{I P P}^{2}$ and the average transmission rate we calculate the ON and OFF periods : $\frac{1}{\sigma_{1}}$ and $\frac{1}{\sigma_{2}}$ respectively. 
In this section we calculate the pdf of the number of bursts during an aggregation period assuming that packets arrive in IPP fashion. We follow the same approach as in the case of Poisson arrivals. We first calculate the MGF of the number of bytes that arrive during a period $T$. Similarly to the Poisson arrival case, we have a random sum of packets whose size is exponentially distributed, and therefore we use equation $5 . M_{B}(t)=M_{N}\left(\ln \left(M_{S}(t)\right)\right)$ where $M_{B}(t)$ is the MGF of the number of bytes during interval $((i-1) T, i T], M_{N}(t)$ is the MGF of the number of packets $N$, and $M_{S}(t)$ the MGF of the packet size. The MGF of the number of packets that arrive during a period $T$ is obtained as follows.

Let: $P_{i j}=\operatorname{Prob}\left\{N_{t}=n, J_{t}=j \mid N_{0}=0, J_{0}=i\right\}$ be the probability that $N_{t}$ arrivals occur during $(0, t]$ given that at time 0 there were 0 arrivals and the IPP was in state $J_{0}=i$ and at time t the IPP was in state $J_{t}=j$. The z-transform of $P_{i j}$ [11] is: $P^{*}(z, t)=e^{(Q-(1-z) \Lambda) t}$ where $Q$ is the infenitesimal generator of the IPP and $\Lambda$ the matrix of arrival rates, i.e.

$$
Q=\left(\begin{array}{cc}
-\sigma_{1} & \sigma_{1} \\
\sigma_{2} & -\sigma_{2}
\end{array}\right), \Lambda=\left(\begin{array}{ll}
\lambda & 0 \\
0 & 0
\end{array}\right)
$$

Now we can use this z-transform to form the generating function of the number of packets. We know that the MGF of a discrete random variable $x$, is its ztransform when $z=e^{s}$. Therefore, $M_{X}(s, t)=P^{*}\left(e^{s}, t\right)$, where $P^{*}(z, t)$ the z-transform of function $P(n, t)$. Thus, the MGF of the number of packets that arrive during $(0, t]$ is: $M_{N}(s, t)=e^{\left(Q-\left(1-e^{s}\right) \Lambda\right) t}$. Substituting $s$ with $\ln \left(M_{S}(-s)\right)$, where $M_{S}(s)=\frac{b}{b+s}$, is the Laplace transform of the exponentially distributed packet size, we obtain:

$$
M_{B}(s, t)=e^{\left(Q-\left(1-\frac{b}{b-s}\right) \Lambda\right) t}
$$

We can now calculate the first two moments $m_{1, I P P}$ and $m_{2, I P P}$ of the number of bytes that arrive during a period $T$, by setting $t=T$. We have:

$$
m_{1, I P P}=\mathbf{e}^{T} \mu(s)=\mathbf{e}^{T} M^{\prime}(T) \mathbf{e}
$$

where: $M^{\prime}(T)=\left[\frac{\partial}{\partial s} M_{B}(s, T)\right]_{s=0}$ and $\mathbf{e}$ is a column vector of 1 's and $\mathbf{e}^{T}$ a row vector of 1 's. In order to differentiate the MGF we use the eigenvalue decomposition of the matrix exponential given in 17. The eigenvalue decomposition always exists for this MGF. We have: $e^{A t}=P e^{D t} P^{-1}$ where $A=\left(Q-\left(1-\frac{b}{b-s}\right) \Lambda\right) t$ $D$ is the diagonal matrix of the eigenvalues of $A, P$ is the matrix composed of eigenvectors and $P^{-1}$ the inverse matrix of $P$. After differentiating and using the chain rule we get:

$$
M^{\prime}(T)=\frac{\partial e^{A T}}{\partial s}=\frac{\partial P}{\partial s} e^{D T} P^{-1}+P e^{D T} \frac{\partial P^{-1}}{\partial s}+T P e^{D T} \frac{\partial D}{\partial s} P^{-1}
$$

Substituting the above in equation 18 we have:

$$
m_{1, I P P}=\pi_{1} \frac{\lambda T}{b}
$$


The above expression is intuitively obvious, as it is the mean duration of the ON period $\pi_{1}$ multiplied by the mean number of bytes that arrive during this period $\frac{\lambda T}{b}$. The second moment is given by:

$$
m_{2, I P P}=\mathbf{e}^{T} \mu_{2}(s)=\mathbf{e}^{T} M^{(2)}(T) \mathbf{e}
$$

where the second derivative $M^{(2)}(T)=\left[\frac{\partial^{2}}{\partial s} M_{B}(s, T)\right]_{s=0}$ is obtained by applying the chain rule to equation 19, We have:

$$
\begin{gathered}
M^{(2)}(T)=\frac{\partial^{2} P}{\partial s} e^{D T} P^{-1}+2 T \frac{\partial P}{\partial s} e^{D T} \frac{\partial D}{\partial s} P^{-1}+2 \frac{\partial P}{\partial s} e^{D T} \frac{\partial P^{-1}}{\partial s} \\
+2 T P e^{D T} \frac{\partial D}{\partial s} \frac{\partial P^{-1}}{\partial s}+P e^{D T} \frac{\partial^{2} P^{-1}}{\partial s}+T^{2} P e^{D T}\left(\frac{\partial D}{\partial s}\right)^{2} P^{-1} \\
+T P e^{D T} \frac{\partial^{2} D}{\partial s} P^{-1}
\end{gathered}
$$

Now we can use the two moments to approximate the pdf $f_{B}(x)$ of the number of bytes that arrive during a period $T$, with a $C_{2}$ distribution as in the previous section.

If $c^{2}<0.5$ then we used the generalized Erlang $k$ approximation, where: $\frac{1}{k} \leq c^{2} \leq \frac{1}{k-1}$ In the generalized Erlang $k$ with probability $a$, after the first exponential phase the service continues for the rest of the $k-1$ stages or it ends with probability $1-a$. Probability $a$ is given by [10]:

$$
1-a=\frac{2 k c^{2}+k-2-\sqrt{k^{2}+4-4 k c^{2}}}{2\left(c^{2}+1\right)(k-1)}
$$

and service rate: $\mu=\frac{1+(k-1) a}{m_{1}}$

The probability of having $\mathrm{k}$ bursts at the end of an aggregation period $T$ is:

$$
P[k \text { bursts }]=\int_{(k-1) B_{\max }}^{k B_{\max }-1} f_{Y}(y), k>=1
$$

where $f_{Y}(y)$ is the pdf of the $C_{2}$ or the generalized Erlang pdf as above. Notice that $B_{\text {min }}=0$ in this model, since there are no residual bytes included. The case of $B_{\min }>0$ can be modelled as in the previous section.

Finally, we note that the number of bytes that arrive during each interval $T$ is approximated by the number of bytes that arrive in $(0, t]$. In reality, the IPP state at the beginning of each interval $T$ is the state at the end of the previous period $T$. In our model, we approximate this by assuming that the IPP is at state $i$ at the beginning of the interval $T$ and then uncondition on this event.

\section{$4 \quad$ Numerical Results}

In this section we compare our approximate analytic results to simulation data for both Poisson and IPP arrivals. A simulation program was writen in C to simulate the behavior of the burst aggregation algorithm under study, with Poisson 

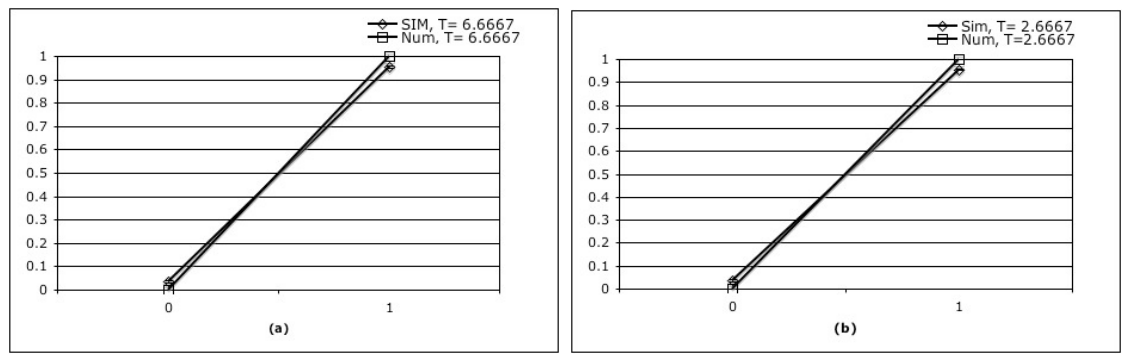

Fig. 1. Probability distribution of the number of bursts. Poisson Arrivals, no residual, $B_{\min }=0$.

and IPP arrivals. 95\% confidence intervals were computed using the method of batch means. The number of batches was fixed to 30 and each batch consisted of 100,000 aggregation periods $T$. The confidence intervals were extremely small and they are not discernible in the figures.

\subsection{Poisson Arrivals}

Figures 1, 2] and 3 give approximation and simulation results of the probability distribution of the number of bursts for the case where $c^{2}>0.5$. In this case the probability distribution is computed using the fitted $C_{2}$ distribution. Figures 1 (a) and 1 (b) give results for $T=6.6667$ and $T=2.6667$ respectively. $B_{\min }=0$, which means that the probability of having zero bursts is $0, B_{\max }=112$ Kbytes.

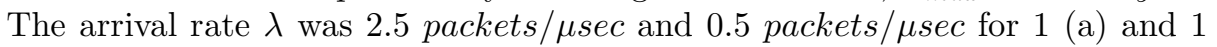
(b) respectively. The average packet size $1 / b$ is obtained from the expression: $1 / \lambda=\frac{8(1 / b)(\text { bits })}{\text { transmission speed }(\text { Gbps })}$, where the transmission speed is 10 Gbps. We observe that the results are quite accurate. In the case where $B_{\min }=16$ Kbytes, we have observed that our approximation is slightly affected by the residual bytes. This is because in the case where $B_{\min }=16$ Kbytes we may have residual bytes but this model does not include them since they are very few and usually 0 . This is why we have a variation from the simulation results.

Figures 2 (a) and2 (b) give the analytical and simulation results of the probability distribution of the number of bursts when $B_{\min }$ is close to $B_{\max }$. In this case, we include the residual bytes from the previous aggregation period in our calculation. $B_{\max }=200$ Kbytes, $B_{\min }=150$ Kbytes, the average packet size $1 / b=125$ Kbytes and transmissionSpeed $=1$ Tbps. These parameters could be meaningful in very high speed networks with dedicated connections where large file transfers may occur, such as in a Grid environment. In Figure 2 (a) and in Figure 2(b) $T=2.05549 \mu \mathrm{sec}$ and $T=2.91172 \mu \mathrm{sec}$. Our approximation is very accurate in this case, only a slight variation is observed for a low number of bursts that could be justified since the assumption that the residual bytes are uniformly distributed in $\left[0, B_{\min }\right)$ is not always accurate. This assumption is more accurate when $B_{\min }$ is higher $(180 \mathrm{Kbytes})$ and therefore the difference $B_{\max }-B_{\min }$ is smaller, as can be viewed in Figures 3 (a) and 3 (b). 

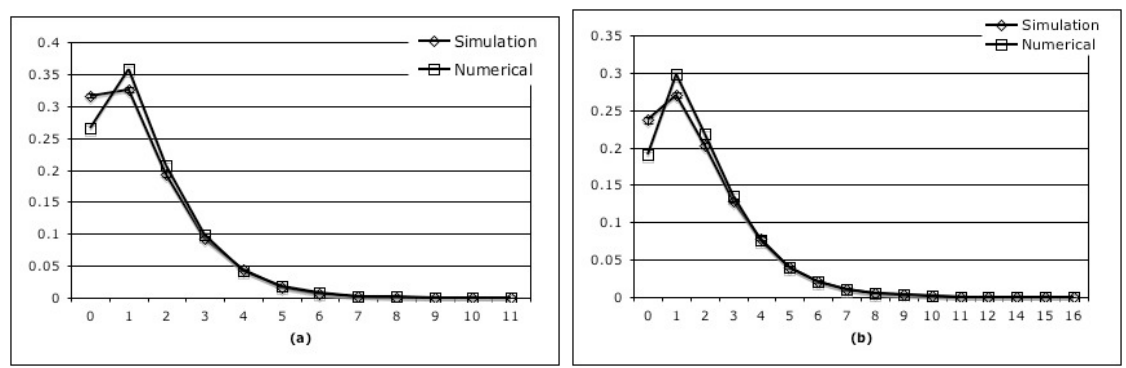

Fig. 2. Probability distribution of the number of bursts. Poisson Arrivals, with residual, $B_{\text {min }}=150$ Kbytes.
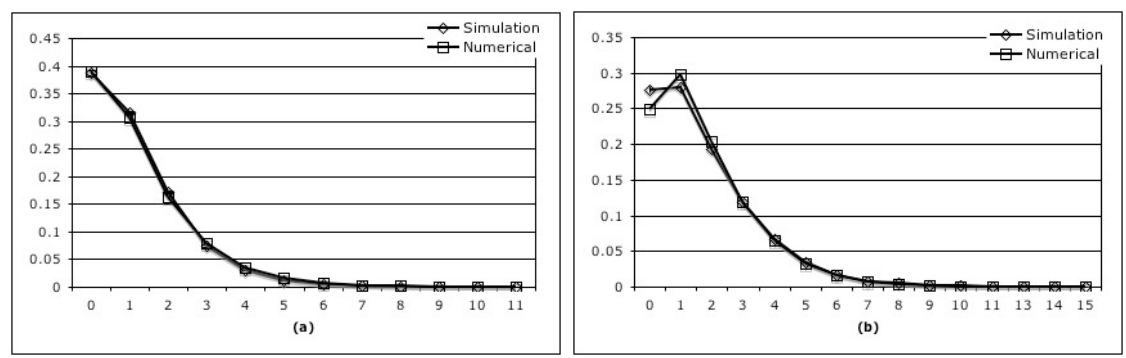

Fig. 3. Probability distribution of the number of bursts. Poisson Arrivals, with residual, $B_{\text {min }}=180$ Kbytes.

Figure 4 gives results for a case where $c^{2}<0.5$. As mentioned above, we analyze this case, either by computing equation 2 for a limited range of values, or using the approximation given in (18). In this case the approximate results match the simulation data. The latter approach is very fast but it does not give accurate results in all cases. The former method is much slower, but gives very accurate results.

Figures 4 (a), 4 (b), 4 (c) and 4 (d) give the analytic results obtained using both methods and the simulation results for $T=128,256,512$ and $1024 \mu \mathrm{sec}$ respectively. No residual is included and $B_{\min }=16$ Kbytes. The transmission speed was $10 \mathrm{Gbps}$, the average packet size, based on IP packets, was 500 bytes. In the case where $T=128,256,512 \mu \mathrm{sec}$ both analytic methods give accurate results. When $T=1024 \mu \mathrm{sec}$ the aggregation period is high and the variability in the number of bursts increases. Thus in this case the approximation method is not accurate. If the residual bytes are included then we have observed that both our analytic models give almost the same results as the simulation model for a variable aggregation period $T$. We note that when $c^{2}<0.5$ the distribution of the available number of bytes at the end of each aggregation period is almost constant. This explains why the probability distribution of the number of bursts is almost constant. The result can prove useful in traffic engineering as it may simplify the architecture. 

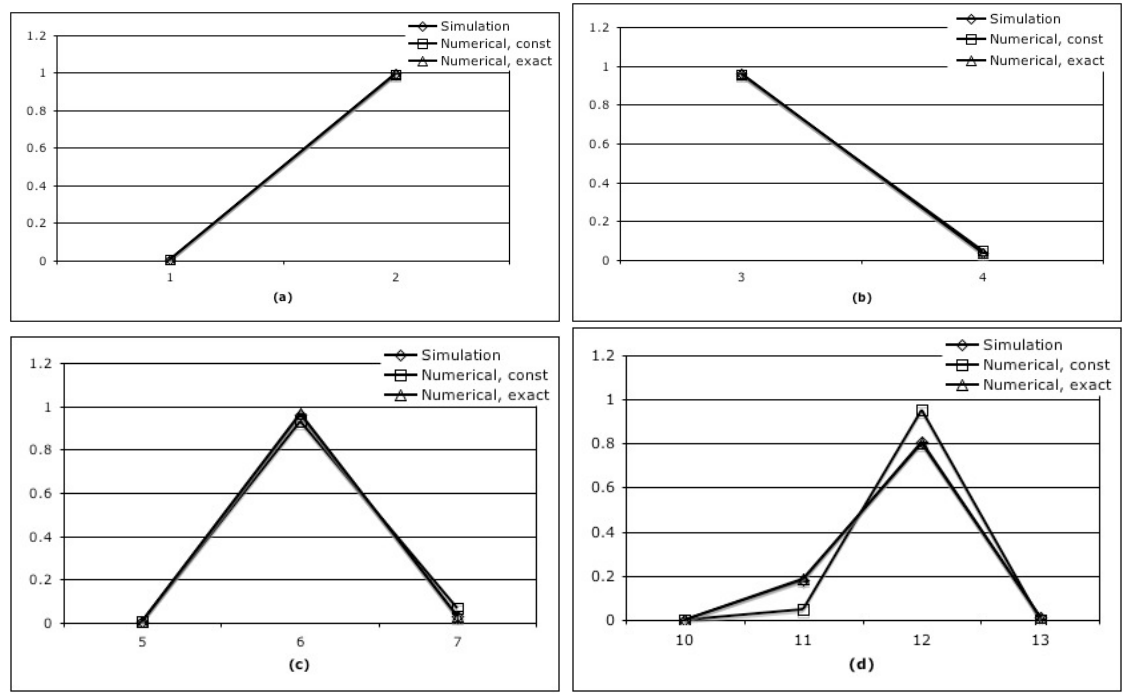

Fig. 4. Probability distribution of the number of bursts. Poisson Arrivals, no residual, $B_{\min }=16$ Kbytes.
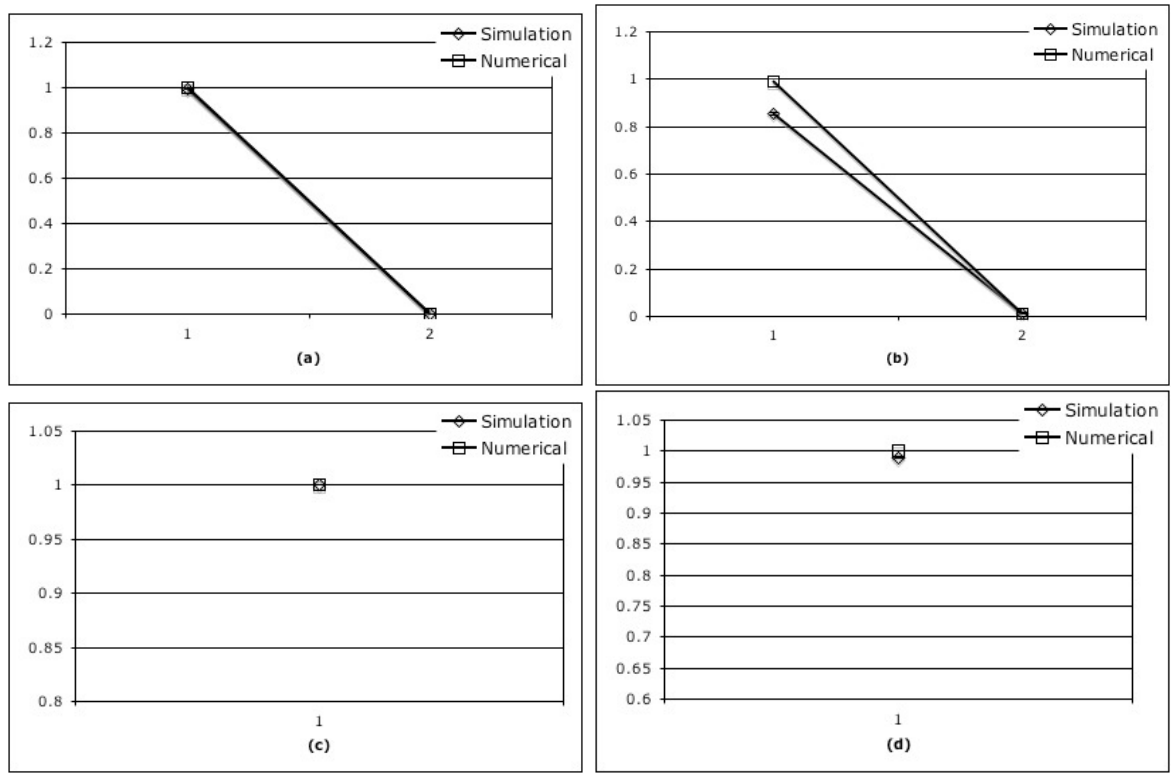

Fig. 5. Probability distribution of the number of bursts. IPP arrivals, no residual, $B_{\text {min }}=0$ bytes, Average Arrival Rate $=1$ Gbps. 


\subsection{IPP Arrivals}

The results that are given in Figure 5 were obtained under the following assumptions: transmission speed $=10 \mathrm{Gbps}$, average transmission rate $=1 \mathrm{Gbps}$, $c_{I P P}^{2}=5, B_{\min }=0, B_{\max }=16$ or 112 Kbytes. average packet size $1 / b=500$ bytes, and arrival rate during ON period: $\lambda=2.5$ packets $/ \mu$ sec. In this case, $c^{2}>0.5$ and we use the $C_{2}$ fit.

Figures 5 (a) and 5 (b) show the probability of having $k$ bursts $B_{\max }=$ 16 Kbytes and the aggregation period is $T=16,32 \mu \mathrm{sec}$ respectively. In Figures 5. (c) and 5 (d) we increase the $B_{\max }$ to 112 Kbytes. There is almost no difference between the simulation results and the numerical results.

\section{Conclusions}

The burst aggregation process defines to a large extent the burst arrival process to the OBS network. This burst arrival process has not as yet been adequately studied. However, it is important that it is well characterized if we are to understand better the performance of the OBS network. In this paper, we have obtained analytically the probability distribution of the number of bursts created by an aggregation algorithm that uses a timer and a minimum and maximum burst size. The analytical results are approximate but they seem to have good accuracy.

Acknowledgements. We would like to thank Boldea Otilia for her helpful comments in the IPP analysis.

\section{References}

1. Xu, L., Perros, H.: Performance analysis of an ingress optical burst switching node. (Submitted. http://www.csc.ncsu.edu/faculty/perros/Xu5.pdf)

2. Yu, X., Li, J., Cao, X., Chen, Y., Qiao, C.: Traffic statistics and performance evaluation in optical burst switched networks. Journal of lightwave technology 22(12) (2004) 2722-2738

3. de Vega Rodrigo, M., Gotz, J.: An analytical study of optical burst switching aggregation strategies. In: Broadnets 2004, IEEE (2004)

4. Hu, G., K., D., Gauger, C.: Does burst assembly really reduce the self-similarity. In: In Proc. of the Optical Fiber Communication Conference (OFC). (2003)

5. Xiong, Y., Vandenhoute, M., Cankaya, H.: Control architecture in optical burst switched wdm networks, in ieee jsac. Volume 18. (2000) 1838-1851

6. Xue, F., Yoo, S.J.B.: Self-similar traffic shaping at the edge router in optical packet-switched networks. In: In Proc. of IEEE International Conference on Communications. (2002)

7. Viniotis, Y.: Probability and Random Processes for Electrical Engineers. McGrawHill (1997)

8. Yates, R.D., Goodman, D.J.: Probability and Stochastic Processes. John Wiley and Sons (1999) 
9. Kleinrock, L.: Queueing Systems, Volume I: Theory. John Wiley and Sons (1975)

10. Perros, H.G.: Queueing Networks with Blocking, Exact and Approximate Solutions. Oxford University Press (1994)

11. Fischer, W., Meier-Hellstern, K.: The Markov-Modulated Poisson Process (MMPP) cookbook. Performance Evaluation 18 (1992) 149-171 